\title{
Stop searches in flavourful supersymmetry
}

\author{
Andreas Crivellin, ${ }^{a, d}$ Ulrich Haisch ${ }^{b, d}$ and Lewis C. Tunstall ${ }^{c}$ \\ ${ }^{a}$ Paul Scherrer Institut, CH-5232 Villigen PSI, Switzerland \\ ${ }^{b}$ Rudolf Peierls Centre for Theoretical Physics, University of Oxford, \\ 1 Keble Road, Oxford OX1 3NP, United Kingdom \\ ${ }^{c}$ Albert Einstein Center for Fundamental Physics, Institute for Theoretical Physics, \\ University of Bern, Sidlerstrasse 5, CH-3012 Bern, Switzerland \\ ${ }^{d}$ CERN, Theory Division, CH-1211 Geneva 23, Switzerland \\ E-mail: andreas.crivellin@cern.ch, ulrich.haisch@physics.ox.ac.uk, \\ tunstall@itp.unibe.ch
}

ABSTRACT: Natural realisations of supersymmetry require light stops $\tilde{t}_{1}$, making them a prime target of LHC searches for physics beyond the Standard Model. Depending on the kinematic region, the main search channels are $\tilde{t}_{1} \rightarrow t \tilde{\chi}_{1}^{0}, \tilde{t}_{1} \rightarrow W b \tilde{\chi}_{1}^{0}$ and $\tilde{t}_{1} \rightarrow c \tilde{\chi}_{1}^{0}$. We first examine the interplay of these decay modes with $\tilde{c}_{1} \rightarrow c \tilde{\chi}_{1}^{0}$ in a model-independent fashion, revealing that a large parameter space region with stop mass values $m_{\tilde{t}_{1}}$ up to $530 \mathrm{GeV}$ is excluded for any $\tilde{t}_{1} \rightarrow c \tilde{\chi}_{1}^{0}$ branching ratio by LHC Run I data. The impact of $\tilde{c}_{1} \rightarrow c \tilde{\chi}_{1}^{0}$ decays is further illustrated for scenarios with stop-scharm mixing in the right-handed sector, where it has previously been observed that the stop mass limits can be significantly weakened for large mixing. Our analysis shows that once the $\tilde{c}_{1} \rightarrow c \tilde{\chi}_{1}^{0}$ bounds are taken into account, non-zero stop-scharm mixing can lead to an increase in the allowed parameter space by at most $35 \%$, with large areas excluded for arbitrary mixing.

KeYwORDS: Supersymmetry Phenomenology

ArXiv EPrint: 1604.00440 


\section{Contents}

1 Introduction 1

2 Stop search combination for $m_{\tilde{t}_{1}}-m_{\tilde{\chi}_{1}^{0}}>m_{t}$

3 Stop search combination for $m_{W}+m_{b}<m_{\tilde{t}_{1}}-m_{\tilde{\chi}_{1}^{0}}<m_{t} \quad 8$

4 Exclusion limits for purely right-handed up-squark mixing 11

5 Conclusions and outlook $\quad 14$

$\begin{array}{ll}\text { A Event generation } & 15\end{array}$

\section{Introduction}

A key feature of supersymmetric extensions of the Standard Model (SM) is the fact that radiative corrections to the Higgs potential can induce electroweak symmetry breaking in a technically natural fashion. Since top quarks and top squarks dominate the radiative corrections, naturalness requires their masses to be of similar magnitudes to ensure a sufficient cancellation of quadratic divergences. Apart from the gluino, Higgsinos and the left-handed bottom squark, the rest of the superpartners are less important for naturalness, and may well have masses above the reach of the LHC [1-7]. A spectrum with the above hierarchy is a typical starting point for phenomenological analyses in supersymmetry (SUSY).

Although light stops are required for naturalness, they can reintroduce fine-tuning in minimal SUSY because the Higgs is typically predicted to be light. For instance, to accommodate a Higgs mass of $125 \mathrm{GeV}$ in the Minimal Supersymmetric SM (MSSM), the stop masses must be around $1 \mathrm{TeV}$, at the cost of tuning at the percent level or worse. Reconciling these two features, light stops for naturalness and heavier stops for the Higgs mass, constitutes the "little hierarchy problem". However, in contrast to naturalness, the little hierarchy problem is model dependent and tightly bound to the MSSM. SUSY models that can generate a sufficiently heavy Higgs with improved naturalness include scenarios with non-decoupling $D$-terms [8] and the next-to-minimal supersymmetric SM with special parameter choices [9].

Naturalness considerations aside, there are additional reasons to expect light stops if SUSY is realised in nature. For instance, the renormalisation group evolution from a high scale with universal squark masses typically drives the masses of the third generation squarks to small values [10]. Light stops also help accommodate the observed dark matter relic density [11, 12] and are an essential ingredient in realising baryogenesis [13-15].

Experimentally, the bounds on the lightest stop mass $m_{\tilde{t}_{1}}$ are much weaker than the limits on the other coloured superpartners, i.e. the squarks of the first two generations and 
the gluino $[16,17]$. There are three main kinematic regions where different channels are used to search for stops, namely

$$
\begin{aligned}
& \text { R1) } m_{\tilde{t}_{1}}-m_{\tilde{\chi}_{1}^{0}}>m_{t}: \tilde{t}_{1} \rightarrow t \tilde{\chi}_{1}^{0}, \\
& \text { R2) } m_{W}+m_{b}<m_{\tilde{t}_{1}}-m_{\tilde{\chi}_{1}^{0}}<m_{t}: \tilde{t}_{1} \rightarrow W b \tilde{\chi}_{1}^{0}, \\
& \text { R3) } m_{c}<m_{\tilde{t}_{1}}-m_{\tilde{\chi}_{1}^{0}}<m_{W}+m_{b}: \tilde{t}_{1} \rightarrow c \tilde{\chi}_{1}^{0} \text { and } \tilde{t}_{1} \rightarrow b f f^{\prime} \tilde{\chi}_{1}^{0} .
\end{aligned}
$$

Here $m_{\tilde{\chi}_{1}^{0}}$ denotes the mass of the lightest neutralino, constituting the lightest superpartner (LSP), while $m_{W}, m_{b}$ and $m_{c}$ are the mass of the $W$ boson, the bottom quark and the charm quark, respectively.

In each region, the results from the ATLAS and CMS searches are interpreted in the context of simplified models, where the branching ratio for each decay mode is fixed to $100 \%$ and flavour violation is assumed to be absent. Under these assumptions, the resulting limits on $m_{\tilde{t}_{1}}$ in the region $\mathrm{R} 1$ are strong, reaching up to stop masses of $715 \mathrm{GeV}$ in the case of ATLAS [18-23] and of almost $800 \mathrm{GeV}$ in the case of CMS [24-28]. It has been observed [29-34], however, that these limits can be weakened if non-minimal sources of flavour violation are present. This occurs because flavour-violating effects enhance the decay width for $\tilde{t}_{1} \rightarrow c \tilde{\chi}_{1}^{0}$, and thereby reduce the branching ratio for $\tilde{t}_{1} \rightarrow t \tilde{\chi}_{1}^{0}$ from unity. On the other hand, if the decay width of $\tilde{t}_{1} \rightarrow c \tilde{\chi}_{1}^{0}$ becomes large, the limits from direct $\tilde{c}_{1}$ pair production and subsequent scharm decay $\tilde{c}_{1} \rightarrow c \tilde{\chi}_{1}^{0}$ [35] become relevant, which apply to $\tilde{t}_{1} \rightarrow c \tilde{\chi}_{1}^{0}$ as well once the branching ratio is large. In the second region $\mathrm{R} 2$, the situation is similar. The limits on $m_{\tilde{t}_{1}}$ reach only up to around $300 \mathrm{GeV}[18,20,24]$ and the three-body decay $\tilde{t}_{1} \rightarrow W b \tilde{\chi}_{1}^{0}$ is suppressed by phase space, so that $\tilde{t}_{1} \rightarrow c \tilde{\chi}_{1}^{0}$ can compete for relatively small off-diagonal elements in the squark mass matrix [36]. Again, once stop-scharm mixing and therefore the decay width for $\tilde{t}_{1} \rightarrow c \tilde{\chi}_{1}^{0}$ is sizeable, searches for charm signatures [37] can become relevant. Finally, in the third region R3, $\tilde{t}_{1} \rightarrow c \tilde{\chi}_{1}^{0}$ is typically the dominant decay mode and the four-body decay $\tilde{t}_{1} \rightarrow b f f^{\prime} \tilde{\chi}_{1}^{0}[20,37]$ can only compete for scenarios resembling Minimal Flavour Violation (MFV) [38].

The purpose of this article is to examine the complementarity of $\tilde{c}_{1} \rightarrow c \tilde{\chi}_{1}^{0}$ searches with the standard channels $\tilde{t}_{1} \rightarrow W b \tilde{\chi}_{1}^{0}$ and $\tilde{t}_{1} \rightarrow c \tilde{\chi}_{1}^{0}$ in the presence of non-minimal sources of flavour violation. In section 2 , we introduce the basic ideas behind our combination procedure and apply it to set model-independent limits on $m_{\tilde{t}_{1}}, m_{\tilde{\chi}_{1}^{0}}$ and the branching ratio of $\tilde{t}_{1} \rightarrow c \tilde{\chi}_{1}^{0}$ in the kinematic region $\mathrm{R} 1$ using ATLAS Run I data. The very same exercise is performed in section 3 for the region R2. Focusing on flavour mixing in the righthanded up-squark sector, which is largely unconstrained by quark flavour observables, we then quantify in section 4 the interplay between the different search strategies. As we are interested in non-MFV scenarios in this article, we use the ATLAS bounds for $\tilde{t}_{1} \rightarrow c \tilde{\chi}_{1}^{0}$ directly in region R3. This allows us to provide interesting exclusions in large parts of the entire $m_{\tilde{t}_{1}}-m_{\tilde{\chi}_{1}^{0}}$ plane. Our conclusions and an outlook are presented in section 5 . In order to make our article self-contained, appendix A provides details on the Monte Carlo (MC) simulations that were used to obtain the numerical results presented in our work. 


\section{Stop search combination for $m_{\tilde{t}_{1}}-m_{\tilde{\chi}_{1}^{0}}>m_{t}$}

In this article we focus on the scenario in which the third generation of squarks is lighter than the first two, with the latter assumed to be degenerate in mass. In principle, there could be a mass splitting between the first two generations [39] which could weaken the collider bounds as well [40]. However, with the recent ATLAS analysis [35] the direct bounds on second generation squarks are now stronger than in [40], while non-degenerate squarks of the first two generations lead to unavoidable bounds from kaon and/or $D$ mixing, which further limit the mass splitting [41]. Moreover, assuming universal soft masses at a high scale leads to quasi-degenerate squarks of the first two generations at the low scale, while only the third one is lighter.

We begin our numerical analysis in the kinematic region $\mathrm{R} 1$. In this region the twobody decay $\tilde{t}_{1} \rightarrow t \tilde{\chi}_{1}^{0}$ dominates unless non-minimal sources of flavour violation in the up-squark sector are present that lead to an appreciable rate for $\tilde{t}_{1} \rightarrow c \tilde{\chi}_{1}^{0}$. As illustrated in figure 1, in such cases one faces three different decay configurations: one that involves two top quarks (configuration 1), one with an intermediate top and a charm quark (configuration 2), and finally one with two charm quarks (configuration 3). Since the final state contains two LSPs in all configurations, the visible decay products will be augmented by large amounts of missing transverse momentum $\left(E_{T, \text { miss }}\right)$.

In order to find combined model-independent limits on $m_{\tilde{t}_{1}}, m_{\tilde{\chi}_{1}^{0}}$ and the branching ratio $\operatorname{Br}\left(\tilde{t}_{1} \rightarrow c \tilde{\chi}_{1}^{0}\right)$ in the region $\mathrm{R} 1$, we employ three different ATLAS searches that are all based on around $20 \mathrm{fb}^{-1}$ of $\sqrt{s}=8 \mathrm{TeV}$ data. Specifically, these are

a) $2 c$-tags $+E_{T, \text { miss }}[35]$ : this ATLAS search is originally designed for the decay configuration 3 in figure 1 . In order to maximise the sensitivity of this search, three distinct signal regions (SRs) called mct150, mct200 and mct250 are defined. In all SRs, events have to have a reconstructed primary vertex consistent with the beam positions and to meet basic quality criteria. Furthermore, events are required to contain no residual electron or muon candidate and at least two jets with radius $R=0.4$ and $p_{T}>130,100 \mathrm{GeV}$ and $|\eta|<2.5$. The multijet background contribution with large $E_{T \text {,miss }}$ is suppressed by requiring a minimum azimuthal separation $\left|\Delta \phi\left(\vec{p}_{T, j_{1,2}, 3}, \vec{p}_{T, \text { miss }}\right)\right|>0.4$ between any of the three leading jets and the $E_{T, \text { miss }}$ direction $\vec{p}_{T, \text { miss }}$. The third jet is exempted from this angular requirement, if it has $p_{T}<50 \mathrm{GeV},|\eta|<2.4$ and less than half of the sum of its track $p_{T}$ is associated with tracks matched to the primary vertex. The two highest- $p_{T}$ jets are required to be identified as arising from a charm quark ( $c$-tagged). The algorithm used in the ATLAS analysis achieves a $c$-tagging efficiency of $20 \%$ with a $b$-jet and light-jet rejection fraction of 8 and 200 (medium operating point) [42]. The $E_{T \text {,miss }}$ selections are $E_{T, \text { miss }}>150 \mathrm{GeV}$ and $E_{T, \text { miss }} / \sum_{i=1,2}\left|p_{T, j_{i}}\right|>1 / 3$. To further discriminate between signal and background the invariant mass of the two $c$-tagged jets has to satisfy $m_{c \bar{c}}>200 \mathrm{GeV}$ and a selection based on the boost-corrected contransverse mass $m_{\mathrm{CT}}$ [43] is employed. Depending on the SR, $m_{\mathrm{CT}}>150 \mathrm{GeV}$, $m_{\mathrm{CT}}>200 \mathrm{GeV}$ or $m_{\mathrm{CT}}>250 \mathrm{GeV}$ is required. 


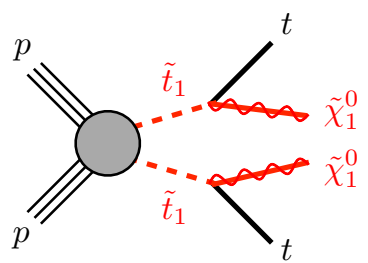

1)

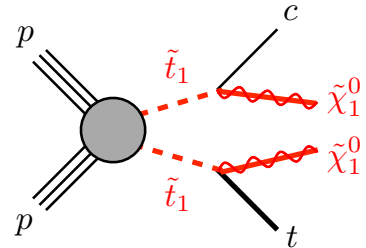

2)

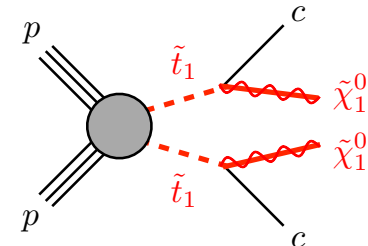

3)

Figure 1. The three different decay configurations relevant for the combination of different stop channels in the kinematic region $\mathrm{R} 1$.

b) 1 lepton +4 jets $+1 b$-tag $+E_{T, \text { miss }}[20]$ : in its original form, this ATLAS search has been tailored for the decay configuration 1 in figure 1 with one top quark decaying hadronically and the other one leptonically. It implements four SRs that target different regions in the $m_{\tilde{t}_{1}}-m_{\tilde{\chi}_{1}^{0}}$ plane and implement different analysis strategies. In our case it turns out that only the SRs called $t N_{-}$diag and $t N \_m e d$ are relevant in the combination. The following preselection criteria are common to the two SRs. Events are required to have a reconstructed primary vertex, $E_{T \text {,miss }}>100 \mathrm{GeV}$, exactly one isolated lepton with $p_{T}>25 \mathrm{GeV}$ and at least four $R=0.4$ jets with $p_{T}>25 \mathrm{GeV}$. Events that do not pass certain data quality requirements are rejected. In the SR tN_diag, the cuts on the three hardest jets are $p_{T}>60,60,40 \mathrm{GeV}$ and $|\eta|<2.5$. The two leading jets have to satisfy $\left|\Delta \phi\left(\vec{p}_{T, j_{1,2}}, \vec{p}_{T, \text { miss }}\right)\right|>0.8$ and at least one jet has to be identified as a bottom-quark jet ( $b$-tagged), assuming an average tagging efficiency of $70 \%[44,45]$. In addition, we require in our analysis $E_{T, \text { miss }}>150 \mathrm{GeV}, E_{T, \text { miss }} / \sqrt{H_{T}}>5 \mathrm{GeV}^{1 / 2}, m_{T}>140 \mathrm{GeV}$, $m_{\text {had-top }} \in[130,205] \mathrm{GeV}$ and impose a veto on loose $\tau$ leptons. Here $H_{T}$ is defined as the scalar $p_{T}$ of the four hardest jets in the event, $m_{T}$ denotes the transverse mass constructed from the lepton transverse momentum and $\vec{p}_{T \text {, miss }}$, while $m_{\text {had-top }}$ represents the hadronic top mass. The selection requirements in tN_med that differ from to that of $\mathrm{tN}_{-}$diag are $p_{T}>80,60,40 \mathrm{GeV}$ for the three leading jets, $\left|\Delta \phi\left(\vec{p}_{T, j_{2}}, \vec{p}_{T, \text { miss }}\right)\right|>0.8, E_{T, \text { miss }}>200 \mathrm{GeV}$ and $m_{\text {had-top }} \in[130,195] \mathrm{GeV}$. A cut on $E_{T, \text { miss }} / \sqrt{H_{T}}$ and a $\tau$-veto is not imposed, but $H_{T \text {,miss }}^{\text {sig }}>12.5$ and $a m_{T 2}>170 \mathrm{GeV}$ is required. Here $H_{T \text {,miss }}^{\text {sig }}$ is an object-based missing transverse momentum that is normalised by the per-event resolution of the jets [20] and $a m_{T 2}$ is an asymmetric variant of the generalised transverse mass [46-49].

c) 6 jets $+2 b$-tags $+E_{T, \text { miss }}[19]$ : this ATLAS search aims to provide the best sensitivity for the decay configuration 1 in figure 1 with both top quarks decaying hadronically. In our analysis, we consider only the SR A1 and the SR A2 out of the possible nine SRs. All events that pass certain quality requirements and do not contain a reconstructed electron or muon with $p_{T}>10 \mathrm{GeV}$ are subjected to the following common selection criteria. They have to have at least six $R=0.4$ jets with $p_{T}>$ $80,80,35,35,35,35 \mathrm{GeV}$ and $|\eta|<2.8$, and out of these jets, two or more have to be $b$-tagged ( $70 \%$ efficiency). The number of events with mismeasured $E_{T \text {,miss }}$ is 
reduced by requiring $\left|\Delta \phi\left(\vec{p}_{T, j_{1,2,3}}, \vec{p}_{T, \text { miss }}\right)\right|>\pi / 5$ and $\left|\Delta \phi\left(\vec{p}_{T, \text { miss }}, \vec{p}_{T, \text { miss }}^{\text {track }}\right)\right|<\pi / 3$, where $\vec{p}_{T, \text { trass }}^{\text {tras }}$ denotes the missing transverse momentum direction determined from the calorimeter system. To further sculpt the signal, the transverse mass calculated from the $b$-tagged jet closest in the azimuthal angle $\phi$ to $\vec{p}_{T \text {,miss }}$ has to satisfy $m_{T}^{b, \text { min }}>175 \mathrm{GeV}$, the mass cuts $m_{b j j}^{0}<225 \mathrm{GeV}$ and $m_{b j j}^{1}<250 \mathrm{GeV}$ on the first and second top candidate [19] are imposed and loose $\tau$ leptons are vetoed. The SRs A1 and A2 only differ in the imposed $E_{T \text {,miss }}$ selection. In the former case, events with $E_{T, \text { miss }}>150 \mathrm{GeV}$ suffice, while in the latter case the stronger requirement $E_{T, \text { miss }}>250 \mathrm{GeV}$ is imposed.

To combine the searches $a, b$ and $c$, we work in the narrow-width approximation and assume that only the decay modes $\tilde{t}_{1} \rightarrow t \tilde{\chi}_{1}^{0}$ and $\tilde{t}_{1} \rightarrow c \tilde{\chi}_{1}^{0}$ are relevant, so that $\operatorname{Br}\left(\tilde{t}_{1} \rightarrow t \tilde{\chi}_{1}^{0}\right)=1-\operatorname{Br}\left(\tilde{t}_{1} \rightarrow c \tilde{\chi}_{1}^{0}\right)$. Both assumptions are satisfied in the kinematic region R1. Using the shorthand notations $\operatorname{Br}=\operatorname{Br}\left(\tilde{t}_{1} \rightarrow c \tilde{\chi}_{1}^{0}\right)$ and $\sigma_{\tilde{t}_{1} \tilde{t}_{1}^{*}}=\sigma\left(p p \rightarrow \tilde{t}_{1} \tilde{t}_{1}^{*}\right)$, the fiducial cross sections $\left(\sigma_{\text {fid }}\right)_{s}$ corresponding to the three different ATLAS searches can then be written in the following way

$$
\begin{aligned}
& \left(\sigma_{\mathrm{fid}}\right)_{a}=\left\{(1-\mathrm{Br})^{2} \epsilon_{1 a}+2 \mathrm{Br}(1-\mathrm{Br}) \epsilon_{2 a}+\mathrm{Br}^{2}\right\} \sigma_{\tilde{t}_{1} \tilde{t}_{1}^{*}}, \\
& \left(\sigma_{\mathrm{fid}}\right)_{b}=\left\{(1-\mathrm{Br})^{2}+2 \mathrm{Br}(1-\mathrm{Br}) \epsilon_{2 b}+\mathrm{Br}^{2} \epsilon_{3 b}\right\} \sigma_{\tilde{t}_{1} \tilde{t}_{1}^{*}}, \\
& \left(\sigma_{\text {fid }}\right)_{c}=\left\{(1-\mathrm{Br})^{2}+2 \operatorname{Br}(1-\mathrm{Br}) \epsilon_{2 c}+\mathrm{Br}^{2} \epsilon_{3 c}\right\} \sigma_{\tilde{t}_{1} \tilde{t}_{1}^{*}} .
\end{aligned}
$$

Here $\epsilon_{t s}$ denotes the efficiency with which the decay configuration $t=1,2,3$ (see figure 1) is detected by the search $s=a, b, c$.

The efficiency maps relevant for the combination of the different stop channels in the region $\mathrm{R} 1$ are displayed in the four panels of figure 2 . They have been obtained by means of the MC simulations described in appendix A. From the plots it is evident that the efficiencies $\epsilon_{t s}$ are not flat, but depend rather sensitively on $m_{\tilde{t}_{1}}$ and $m_{\tilde{\chi}_{1}^{0}}$. This behaviour is expected because changing the mass of the lightest stop and the LSP will modify the kinematic distributions of the final-state particles, which in turn leads to different signal acceptances in the various SRs. In fact, a qualitative understanding of the obtained efficiencies is possible by studying the cutflow of the analysis $a, b$ and $c$ for the different signal configurations 1,2 and 3 . We start by discussing the efficiencies $\epsilon_{1 a}$ and $\epsilon_{2 a}$ shown in the upper left and upper right panel of figure 2, respectively. The first observation is that in most parts of the $m_{\tilde{t}_{1}}-m_{\tilde{\chi}_{1}^{0}}$ plane, the efficiency $\epsilon_{1 a}$ is smaller than $\epsilon_{2 a}$. This is readily understood by recalling from figure 1 that configuration 1 (2) leads to a final state with two bottom quarks (one bottom quark). In the former case, two $b$ quarks have to be misidentified as $c$-jets in order to produce an event in the SRs of search $a$, while in the latter case one mis-tag is sufficient. Another feature that is evident from the plots is that the efficiencies $\epsilon_{1 a}$ and $\epsilon_{2 a}$ both decrease if one approaches the kinematic boundary of region R1. This is due to the fact that the decay chain $\tilde{t}_{1} \rightarrow t \tilde{\chi}_{1}^{0} \rightarrow W^{+} b \chi_{1}^{0}$ and its conjugate will not give rise to significant $E_{T \text {, miss }}$ if the mass difference $m_{\tilde{t}_{1}}-m_{\tilde{\chi}_{1}^{0}}$ is close to $m_{t}$, and as a result the corresponding event is less likely to pass the $E_{T \text {,miss }}$ requirements that are imposed in the scalar charm search $a$. 

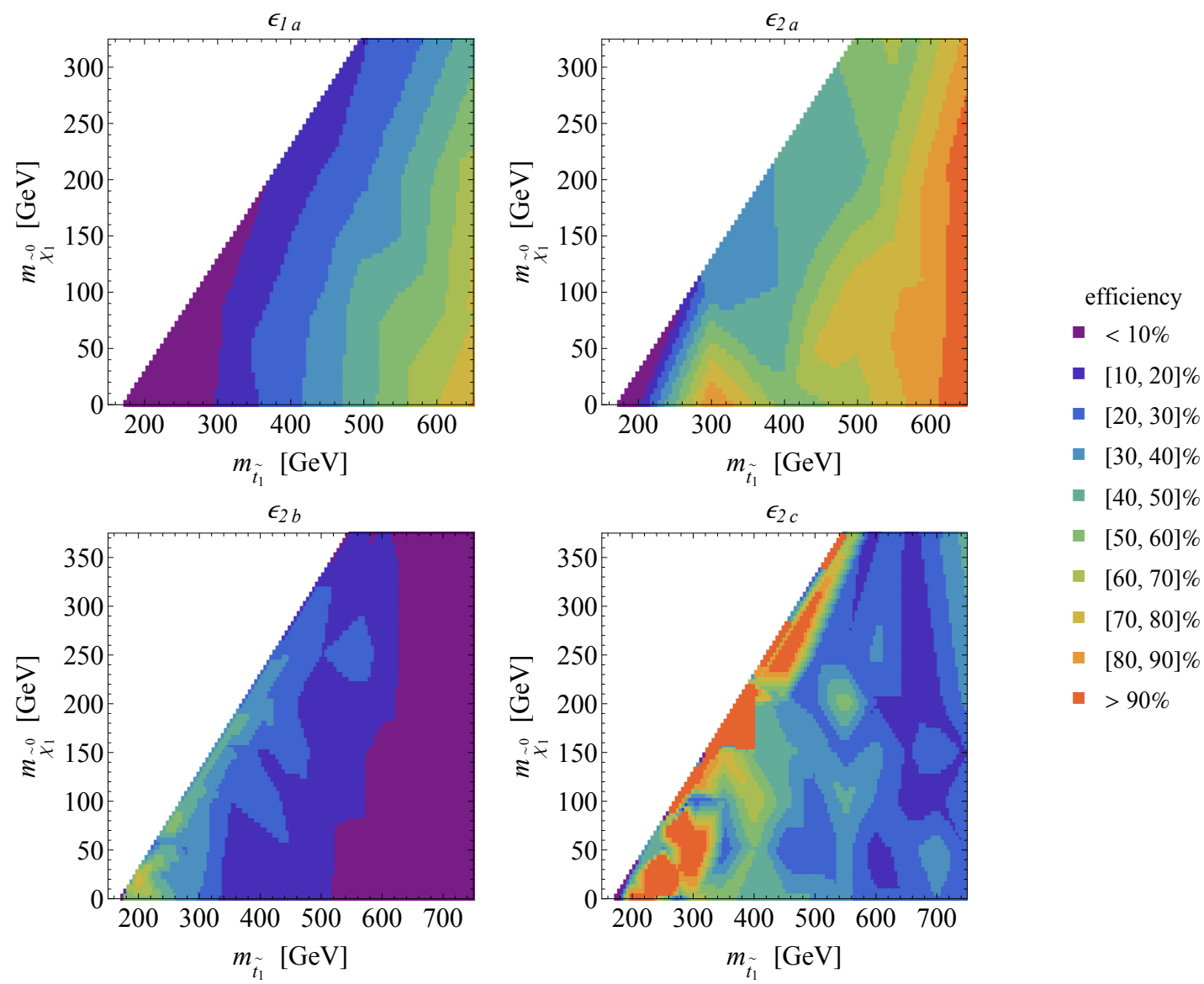

Figure 2. Efficiency maps relevant for the combination of different stop channels in the kinematic region R1. Only the non-trivial efficiencies $\epsilon_{1 a}$ (upper left panel), $\epsilon_{2 a}$ (upper right panel), $\epsilon_{2 b}$ (lower left panel) and $\epsilon_{2 c}$ (lower right panel) are shown.

Simple qualitative explanations of the efficiency maps $\epsilon_{2 b}$ and $\epsilon_{2 c}$ presented in the lower left and lower right panel of figure 2 can also be given. In the case of $\epsilon_{2 b}$, the requirement to have an isolated lepton strongly suppresses the acceptance for final states arising from the configuration 2 , which involves both a $\tilde{t}_{1} \rightarrow t \tilde{\chi}_{1}^{0} \rightarrow W^{+} b \chi_{1}^{0}$ and a $\tilde{t}_{1}^{*} \rightarrow$ $\bar{c} \tilde{\chi}_{1}^{0}$ decay or the combination of charge-conjugated processes. For these decays, the fact that a lepton with $p_{T}>25 \mathrm{GeV}$ can only arise from a leptonic decay of a $W$ boson also explains the finding that $\epsilon_{3 b} \simeq 0$ in the whole kinematic region R1. That $\epsilon_{2 b}$ increases when approaching the kinematic boundary $m_{\tilde{t}_{1}}-m_{\tilde{\chi}_{1}^{0}}=m_{t}$ has to do with the fact that this efficiency is defined relative to the acceptance corresponding to a signal from the configuration 1 in the search $b$ (see (2.1)). The latter acceptance is however suppressed close to the kinematic boundary because there is only little $E_{T, \text { miss }}$ available. Similar arguments hold for $\epsilon_{2 c}$. In this case the requirement that events have to contain two $b$ tags plays the role that the single-lepton tag played before. In fact, events resulting from configuration 2 can end up in the SR, if the final-state charm quark is erroneously $b$-tagged. The corresponding probability is non-negligible and taking into account that the relative 

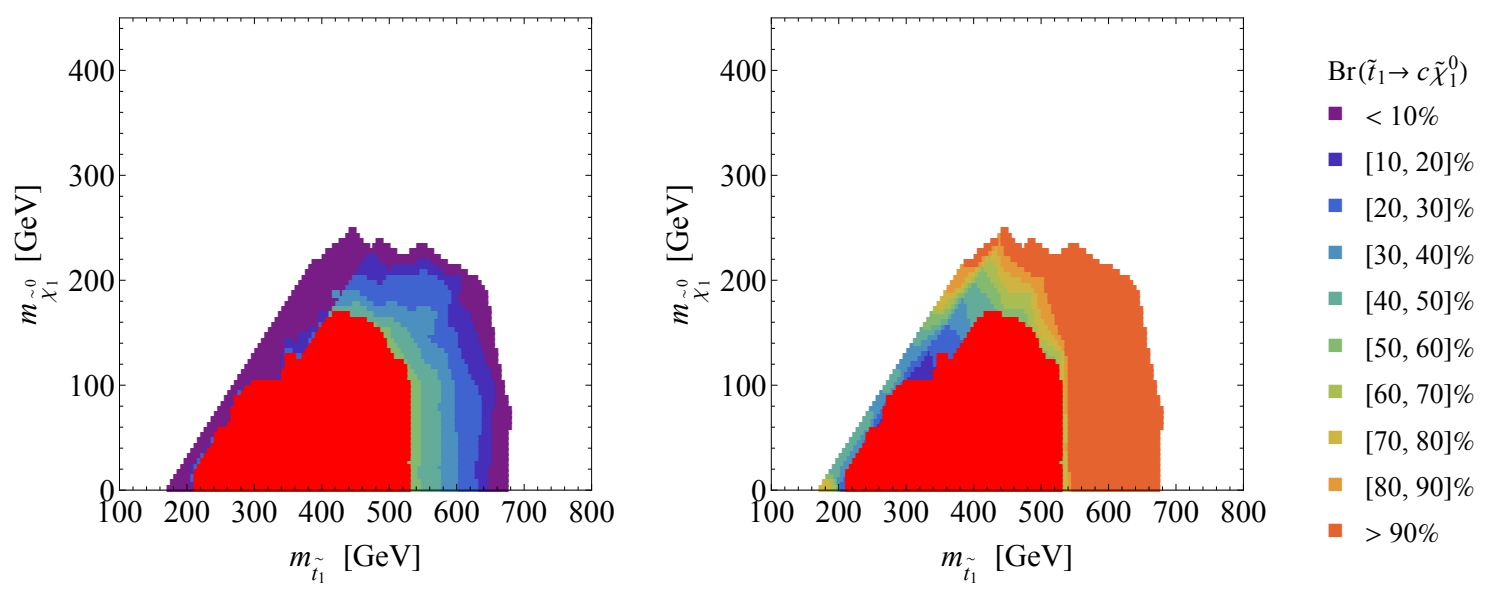

Figure 3. Lower (left panel) and upper (right panel) model-independent limit on $\operatorname{Br}\left(\tilde{t}_{1} \rightarrow c \tilde{\chi}_{1}^{0}\right)$ in the part of the $m_{\tilde{t}_{1}}-m_{\tilde{\chi}_{1}^{0}}$ plane corresponding to the kinematic region R1. The regions coloured red are excluded at $95 \%$ CL for any value of the $\tilde{t}_{1} \rightarrow c \tilde{\chi}_{1}^{0}$ branching ratio.

acceptance for detecting the configuration 2 in the search $c$ again increases for decreasing mass splitting $m_{\tilde{t}_{1}}-m_{\tilde{\chi}_{1}^{0}}$, one obtains numerically $\epsilon_{2 c} \simeq 1$ for points with $m_{\tilde{t}_{1}}-m_{\tilde{\chi}_{1}^{0}}$ not too far from $m_{t}$. Since the probability to mistake two charm quarks for two $b$-jets is essentially zero, we furthermore find that $\epsilon_{3 c} \simeq 0$ for all points of interest in the $m_{\tilde{t}_{1}}-m_{\tilde{\chi}_{1}^{0}}$ plane.

With the efficiency maps at hand, one can then use (2.1) and combine the individual searches to obtain model-independent exclusion limits on $\operatorname{Br}\left(\tilde{t}_{1} \rightarrow c \tilde{\chi}_{1}^{0}\right)$. The outcome of such an exercise is shown in figure 3 . The red region in both panels is excluded at $95 \%$ confidence level (CL) for any value of the $\tilde{t}_{1} \rightarrow c \tilde{\chi}_{1}^{0}$ branching ratio. We observe that depending on the LSP (stop) mass, values of $m_{\tilde{t}_{1}}$ up to $530 \mathrm{GeV}\left(m_{\tilde{\chi}_{1}^{0}}\right.$ up to $160 \mathrm{GeV}$ ) are ruled out by our combination of ATLAS Run I data. Notice that our bound of $530 \mathrm{GeV}$ is only about $35 \%$ weaker than the limit of $715 \mathrm{GeV}$ that ATLAS obtains from a combination of LHC Run I data [21] assuming no stop-scharm mixing. Outside the excluded region our procedure can be used to set lower and upper model-independent limits on $\operatorname{Br}\left(\tilde{t}_{1} \rightarrow c \tilde{\chi}_{1}^{0}\right)$ as indicated by the coloured contours in the left and right panel of the figure. This information will be used in section 4 to put bounds in the $m_{\tilde{t}_{1}}-m_{\tilde{\chi}_{1}^{0}}$ plane for the case of the MSSM with a bino-like LSP and purely right-handed stop-scharm mixing.

It is also interesting to quantify the impact that each individual search has in the combination that leads to the final 95\% CL exclusion limit. For the kinematic region R1, we illustrate the power of the different searches in the left panel of figure 4 . The naive combination corresponds to the choice $\epsilon_{t s}=0$ in (2.1) and is indicated by the dark red contour in the figure. We see that a successive inclusion of the searches $a, b$ and $c$ enlarges the excluded area in the $m_{\tilde{t}_{1}}-m_{\tilde{\chi}_{1}^{0}}$ plane considerably. In fact, it is evident from the three additional red contours that the inclusion of search $a$ has the most pronounced effect in the combination, while adding searches $b$ and $c$ to the mix leads to either no or only a minor improvement of the exclusion limits in the $m_{\tilde{t}_{1}}-m_{\tilde{\chi}_{1}^{0}}$ plane. This feature nicely illustrates one of the main findings of our work, i.e. the observation that the recent ATLAS search 

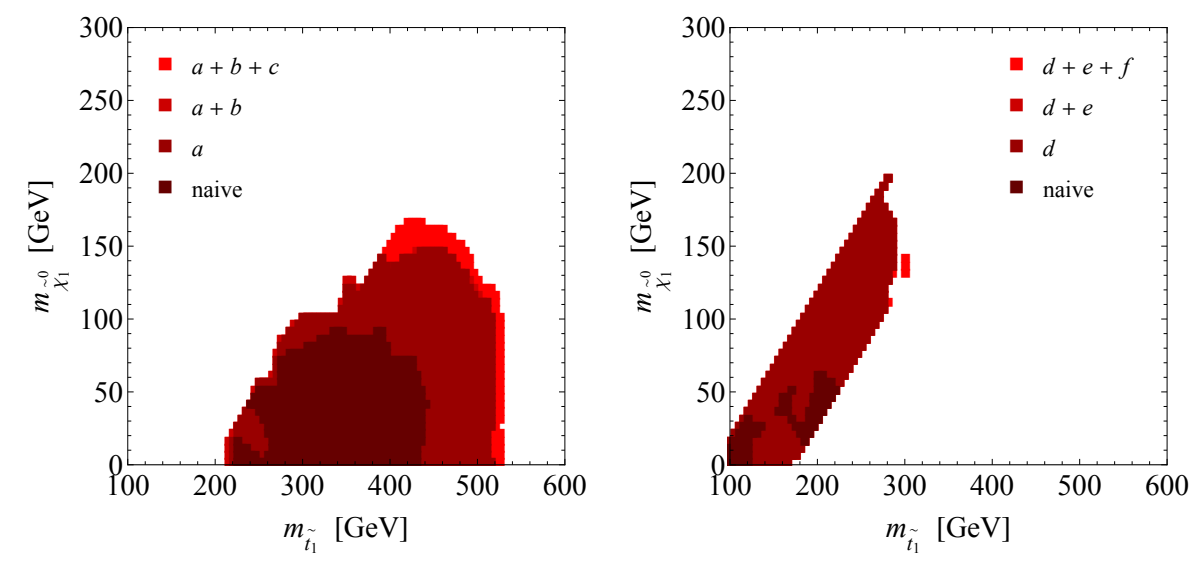

Figure 4. Comparison of the impact of the different searches strategies on the 95\% CL exclusion regions in the $m_{\tilde{t}_{1}}-m_{\tilde{\chi}_{1}^{0}}$ plane. The left (right) panel displays the results of a naive combination and a successive inclusion of the searches $a, b$ and $c(d, e$ and $f)$.

for $\tilde{c}_{1} \rightarrow c \tilde{\chi}_{1}^{0}[35]$ can be recast as a search for $\tilde{t}_{1} \rightarrow c \tilde{\chi}_{1}^{0}$, and that this procedure can be used to set stringent bounds on $m_{\tilde{t}_{1}}$ and $m_{\tilde{\chi}_{1}^{0}}$ in models with non-minimal flavour mixing in the up-squark sector.

\section{Stop search combination for $m_{W}+m_{b}<m_{\tilde{t}_{1}}-m_{\tilde{\chi}_{1}^{0}}<m_{t}$}

We now turn our attention to the kinematic region R2. If stop-scharm mixing is present, both the two-body decay $\tilde{t}_{1} \rightarrow c \tilde{\chi}_{1}^{0}$ and the three-body decay $\tilde{t}_{1} \rightarrow W b \tilde{\chi}_{1}^{0}$ can be phenomenologically relevant. As a result, the final states emerging from two stop decays can contain either two charm quarks (configuration 3), two bottom quarks (configuration 4) or one charm quark and one bottom quark (configuration 5). The additional decay configurations with bottom quarks are depicted in figure 5 . As indicated by the small grey blobs in this figure, the $\tilde{t}_{1} \rightarrow W b \tilde{\chi}_{1}^{0}$ transitions proceeds through an effective four-point vertex which involves the exchange of off-shell particles. In our analysis, we include for simplicity only top-quark exchange, but neglect chargino and sbottom contributions, assuming that these states are sufficiently heavy and decoupled from the spectrum.

In the kinematic region R2, we constrain the $m_{\tilde{t}_{1}}-m_{\tilde{\chi}_{1}^{0}}$ parameter space by again combining three different ATLAS analyses. They are all based on $20.3 \mathrm{fb}^{-1}$ of total integrated luminosity collected at $8 \mathrm{TeV}$ centre-of-mass energy, and implement the following search strategies:

d) 4 jets $+c$-tags $+E_{T \text {,miss }}[37]$ : originally, this ATLAS search has been designed to gain sensitivity to the decay configuration 3 in figure 1 . In our analysis, we consider the $c$-tagged selections $\mathrm{C} 1$ and $\mathrm{C} 2$. The events have to meet basic quality criteria and are vetoed if they contain isolated muons or isolated electrons with $p_{T}>10 \mathrm{GeV}$. As a further preselection $E_{T, \text { miss }}>150 \mathrm{GeV}$ and least one $R=0.4$ jet with $p_{T}>150 \mathrm{GeV}$ and $|\eta|<2.5$ in the final state is required. To be contained in the 


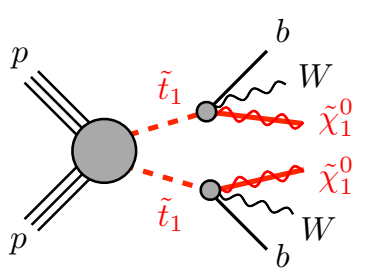

4)

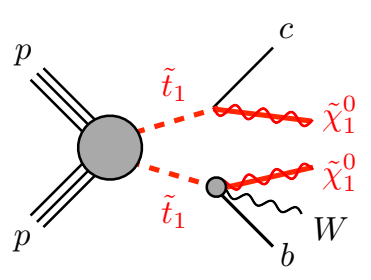

5)

Figure 5. The two additional decay configurations that are relevant for the combination of different stop searches in the kinematic region $\mathrm{R} 2$.

SRs, the events are required to have at least four jets with $p_{T}>30 \mathrm{GeV},|\eta|<2.5$ and $\left|\Delta \phi\left(\vec{p}_{T, j}, \vec{p}_{T, \text { miss }}\right)\right|>0.4$. A $b$-jet veto (2.5 rejection factor) is applied to the selected jets by using a loose $c$-tag requirement (95\% efficiency) [42]. In addition, at least one of the three subleading jets has to pass the medium $c$-tag criteria mentioned earlier in the description of search $a$. The leading jet is then required to have $p_{T}>290 \mathrm{GeV}$ and the two SRs C1 and C2 are defined with $E_{T, \text { miss }}>250 \mathrm{GeV}$ and $E_{T, \text { miss }}>350 \mathrm{GeV}$, respectively.

e) 2 leptons + jets $+E_{T \text {,miss }}[18]$ : this ATLAS search targets the decay configuration 4 in figure 5 with both $W$ bosons decaying leptonically. Our analysis includes the SR L90 and L100 of this search. After passing certain quality requirements, events are preselected if they have exactly two oppositely charged leptons (muons, electrons or one charged lepton of each flavour). At least one of these leptons must have $p_{T}>$ $25 \mathrm{GeV}$ and the invariant mass of the lepton pair has to satisfy $m_{l l}>20 \mathrm{GeV}$. After applying these preselections, events with $m_{l l} \in[71,111] \mathrm{GeV},\left|\Delta \phi\left(\vec{p}_{T, j}, \vec{p}_{T, \text { miss }}\right)\right|>$ 1 , where $j$ denotes the jet closest to the $E_{T, \text { miss }}$ direction, and $\left|\Delta \phi\left(\vec{p}_{T, l l b}, \vec{p}_{T, \text { miss }}\right)\right|<$ 1.5 with $\vec{p}_{T, l l b}=\vec{p}_{T, l_{1}}+\vec{p}_{T, l_{2}}+\vec{p}_{T, \text { miss }}$ are rejected. The SR L90 requires $m_{\mathrm{T} 2}>$ $90 \mathrm{GeV}$ but has no jet requirement, while L100 has a tight jet selection with at least two $R=0.4$ jets with $p_{T}>100,50 \mathrm{GeV}$ and $|\eta|<2.5$. Furthermore, the cut $m_{\mathrm{T} 2}>100 \mathrm{GeV}$ on the lepton-based stransverse mass $[50,51]$ is set in L100.

f) 1 lepton +3 jets $+b$-veto $+E_{T \text {,miss }}[20]$ : at the outset, this ATLAS search is intended for the decay configuration 4 in figure 5 with one leptonic and one hadronic $W$ boson decay. In our combination we use the bcC_diag SR of this analysis, which requires one central lepton with $p_{T}>25 \mathrm{GeV}$ and $|\eta|<1.2$ as well as at least three $R=0.4$ jets with $p_{T}>80,40,30 \mathrm{GeV}$ and $|\eta|<2.5$. Out of the three jets, none are allowed to be $b$-tagged (70\% efficiency) and the two hardest jets have to satisfy $\left|\Delta \phi\left(\vec{p}_{T, j_{1,2}}, \vec{p}_{T, \text { miss }}\right)\right|>2.0,0.8$. The other cuts in our analysis are $E_{T, \text { miss }}>$ $140 \mathrm{GeV}, E_{T, \text { miss }} / \sqrt{H_{T}}>5 \mathrm{GeV}^{1 / 2}, m_{T}>120 \mathrm{GeV}$ and $\Delta R\left(\vec{p}_{T, l}, \vec{p}_{T, j_{1}}\right) \in[0.8,2.4]$. The angular separation in the $\eta-\phi$ plane is defined as $\Delta R=\sqrt{(\Delta \eta)^{2}+(\Delta \phi)^{2}}$.

Under the assumption that only the decay modes $\tilde{t}_{1} \rightarrow W b \tilde{\chi}_{1}^{0}$ and $\tilde{t}_{1} \rightarrow c \tilde{\chi}_{1}^{0}$ are relevant in region $\mathrm{R} 2$, the searches $d, e$ and $f$ can then be combined by using formulas analogous to those presented in (2.1). The corresponding efficiency maps are depicted in figure 6 . In the 

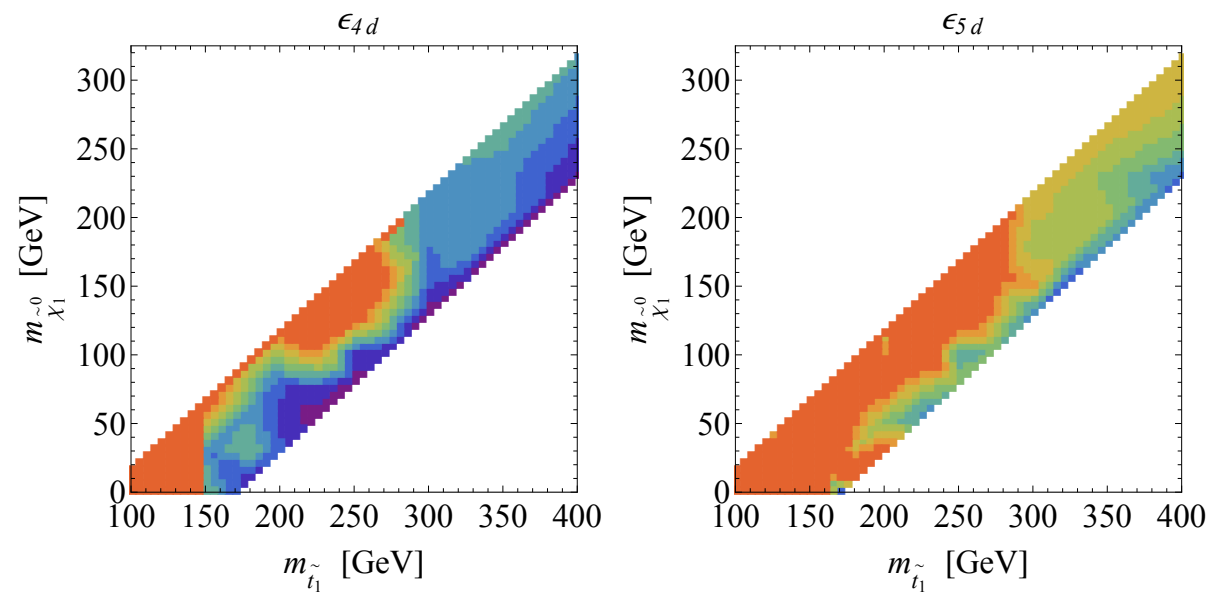

efficiency

- $<10 \%$

- $[10,20] \%$

- $[20,30] \%$

- $[30,40] \%$
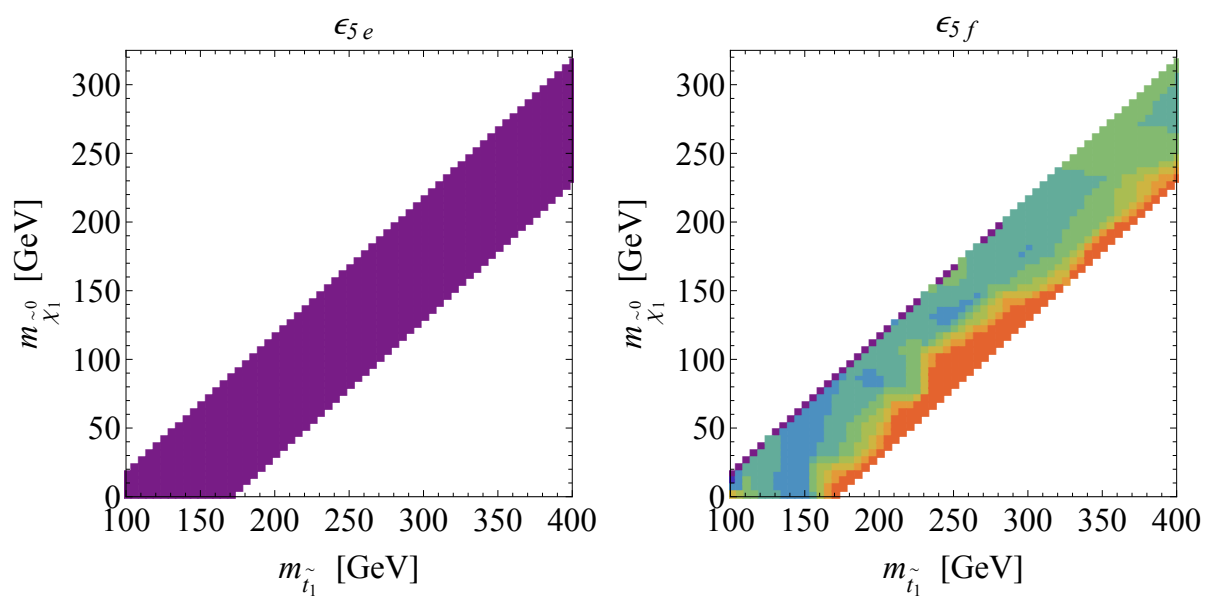

- $[40,50] \%$

- $[50,60] \%$

- $[60,70] \%$

- $[70,80] \%$

- $[80,90] \%$

- $>90 \%$

Figure 6. The efficiencies $\epsilon_{4 d}$ (upper left panel), $\epsilon_{5 d}$ (upper right panel), $\epsilon_{5 e}$ (lower left panel) and $\epsilon_{5 f}$ (lower right panel) relevant for the combination of different stop channels in region R2.

upper left and right panel we show the efficiencies for detecting final states with two bottom quarks (configuration 4) or one bottom and one charm quark (configuration 5) by means of the search $d$. As expected the efficiency $\epsilon_{4 d}$ is typically smaller than $\epsilon_{5 d}$, because the search $d$ involves a $c$-tagged selection. Another noticeable feature of the latter efficiencies is that they are enhanced close to the kinematic boundary $m_{W}+m_{b}=m_{\tilde{t}_{1}}-m_{\tilde{\chi}_{1}^{0}}$ for relatively light stops with $m_{\tilde{t}_{1}} \lesssim 250 \mathrm{GeV}$. This is related to the fact that, compared to $\tilde{t}_{1} \rightarrow c \tilde{\chi}_{1}^{0}$, the decay $\tilde{t}_{1} \rightarrow W b \tilde{\chi}_{1}^{0}$ produces a harder $E_{T, \text { miss }}$ spectrum for masses in this region of the $m_{\tilde{t}_{1}}-m_{\tilde{\chi}_{1}^{0}}$ plane. As a result, events resulting from configuration 4 or 5 more easily pass the $E_{T \text {,miss }}$ requirement of search $d$ than final states arising from configuration 3 .

In the lower left panel of figure 6 , we find that the efficiency $\epsilon_{5 e} \simeq 0$ in the entire R2 region, as a result of the requirement of search $e$ to have two charged leptons in each event. The very same requirement also leads to $\epsilon_{3 e} \simeq 0$. The efficiency map for $\epsilon_{5 f}$ is shown in the lower right panel of the latter figure. One observes that $\epsilon_{5 f}$ grows towards masses satisfying $m_{\tilde{t}_{1}}-m_{\tilde{\chi}_{1}^{0}}=m_{t}$. This feature originates mostly from the fact that for $m_{\tilde{t}_{1}}$ and $m_{\tilde{\chi}_{1}^{0}}$ values close to the boundary between the regions $\mathrm{R} 1$ and $\mathrm{R} 2$, the leading jet arising 

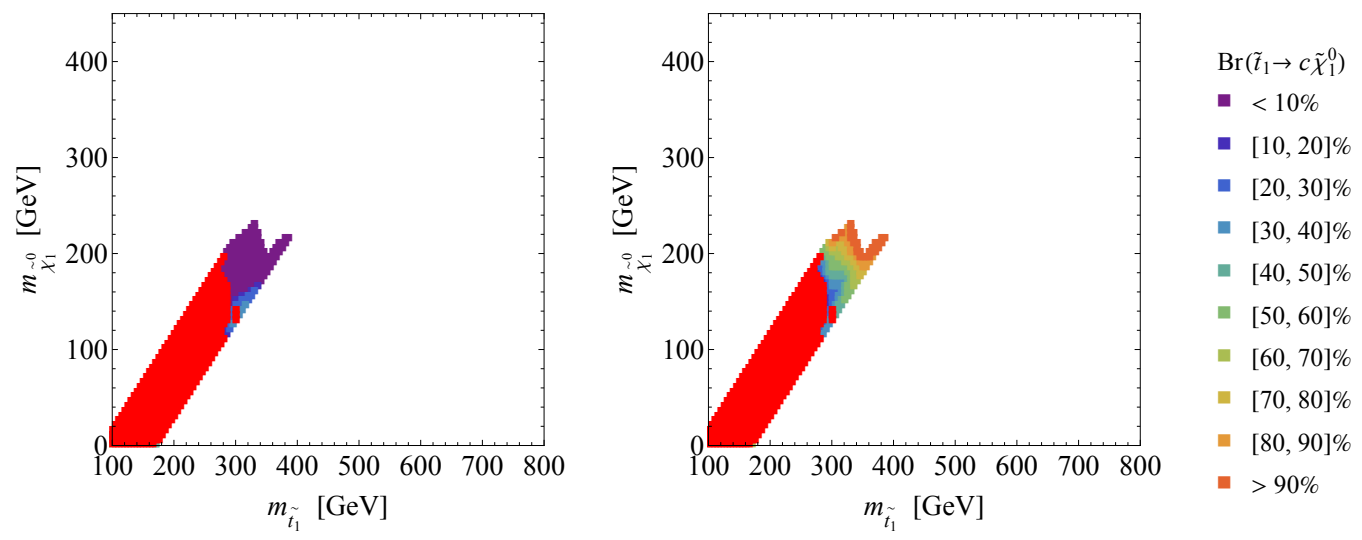

Figure 7. Model-independent lower (left panel) and upper (right panel) limits on $\operatorname{Br}\left(\tilde{t}_{1} \rightarrow c \tilde{\chi}_{1}^{0}\right)$ corresponding to $m_{\tilde{t}_{1}}$ and $m_{\tilde{\chi}_{1}^{0}}$ values in the kinematic region R2. The regions that are excluded at $95 \% \mathrm{CL}$ for any value of the $\tilde{t}_{1} \rightarrow c \tilde{\chi}_{1}^{0}$ branching ratio are coloured red.

from the configuration $f$ is on average harder compared to situations where the masses are close to $m_{W}+m_{b}=m_{\tilde{t}_{1}}-m_{\tilde{\chi}_{1}^{0}}$. As a result of the central lepton requirement, we also find that $\epsilon_{3 f} \simeq 0$ in the full R2 part of the $m_{\tilde{t}_{1}}-m_{\tilde{\chi}_{1}^{0}}$ plane.

In figure 7 , we show the results of our combination procedure in the case of the kinematic region R2. The parameter space that is excluded at 95\% CL for any value of the $\tilde{t}_{1} \rightarrow c \tilde{\chi}_{1}^{0}$ branching ratio is shaded red in both panels, while the coloured contours indicate the model-independent limits on $\operatorname{Br}\left(\tilde{t}_{1} \rightarrow c \tilde{\chi}_{1}^{0}\right)$. We see that $m_{\tilde{t}_{1}}$ values up to around $300 \mathrm{GeV}$ are ruled out by the combined ATLAS Run I data for essentially all LSP masses $m_{\tilde{\chi}_{1}^{0}}$ satisfying $m_{W}+m_{b}<m_{\tilde{t}_{1}}-m_{\tilde{\chi}_{1}^{0}}<m_{t}$. The obtained model-independent bounds on the $\tilde{t}_{1} \rightarrow c \tilde{\chi}_{1}^{0}$ branching ratio will be used in the next section to set limits in the $m_{\tilde{t}_{1}}-m_{\tilde{\chi}_{1}^{0}}$ plane for MSSM scenarios with a bino-like LSP and purely right-handed stop-scharm mixing.

As in section 2, we finally discuss the weight that each search has in our combination. The right panel of figure 4 shows the $95 \%$ CL exclusion contours in the kinematic region R2 that are obtained from a naive combination as well as from a successive inclusion of the searches $d, e$ and $f$. From the figure it is clear that the search $d$ has by far the strongest effect, and that adding the searches $e$ and $f$ does not significantly improve the exclusion in the $m_{\tilde{t}_{1}}-m_{\tilde{\chi}_{1}^{0}}$ plane. This again shows that $c$-tagged SUSY searches, like for instance the ATLAS analysis [37], can also be used to set stringent constraints on $m_{\tilde{t}_{1}}$ and $m_{\tilde{\chi}_{1}^{0}}$ even outside the kinematic region that the search was initially designed to cover.

\section{Exclusion limits for purely right-handed up-squark mixing}

In order to illustrate the effects of flavour mixing in the up-squark sector, we consider a simplified model consisting of a bino-like LSP and a purely right-handed top-like squark $\tilde{t}_{1}$ that is an admixture of $\tilde{t}_{R}$ and $\tilde{c}_{R}$ flavour eigenstates. Since $\tilde{t}_{R}-\tilde{c}_{R}$ mixing is induced by flavour non-diagonal entries in the squark mass-squared matrix $M_{\tilde{u}}^{2}$, it is convenient to use 

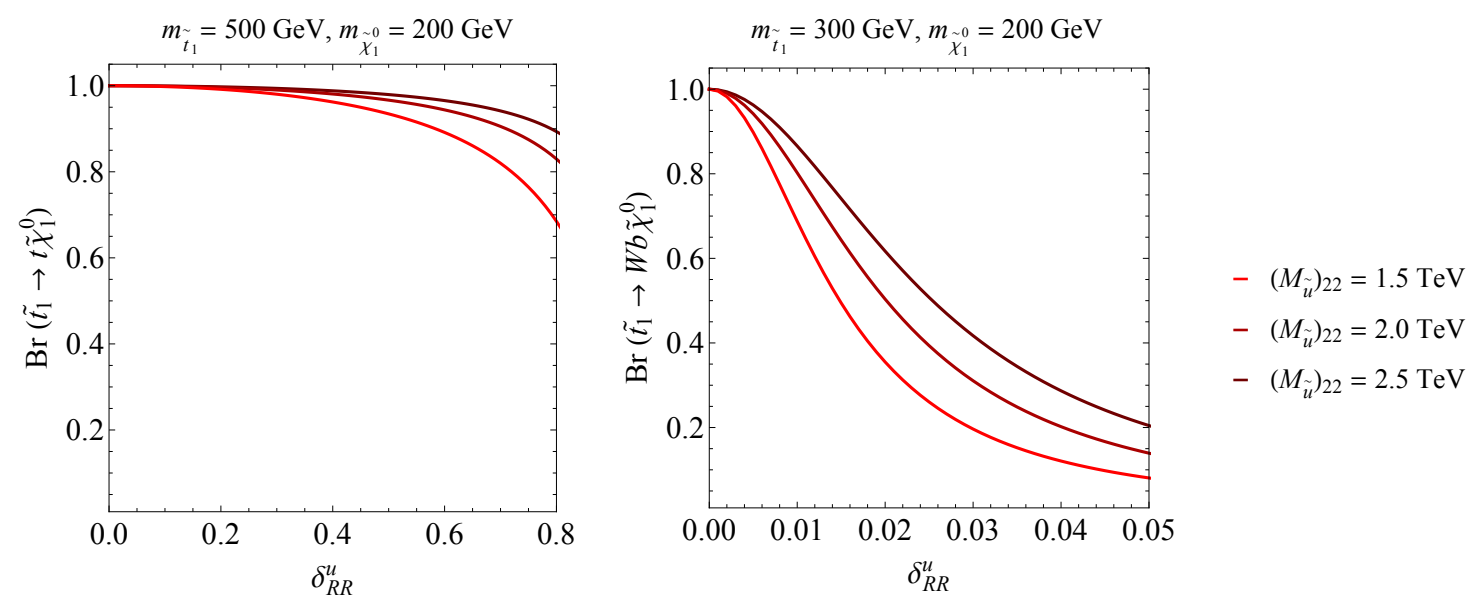

Figure 8. The branching ratios $\operatorname{Br}\left(\tilde{t}_{1} \rightarrow t \tilde{\chi}_{1}^{0}\right)$ (left panel) and $\operatorname{Br}\left(\tilde{t}_{1} \rightarrow W b \tilde{\chi}_{1}^{0}\right)$ (right panel) as a function of the mass insertion parameter $\delta_{R R}^{u}$. The lightest stop mass and the LSP mass have been fixed to $m_{\tilde{t}_{1}}=500 \mathrm{GeV}$ and $m_{\tilde{\chi}_{1}^{0}}=200 \mathrm{GeV}\left(m_{\tilde{t}_{1}}=300 \mathrm{GeV}\right.$ and $\left.m_{\tilde{\chi}_{1}^{0}}=200 \mathrm{GeV}\right)$ to obtain the left (right) plot. The different curves in the panels correspond to different choices of $\left(M_{\tilde{u}}\right)_{22}$ as indicated by the legend on the right-hand side in the figure.

the mass insertion method [52] to express flavour constraints in terms of the dimensionless quantity

$$
\delta_{R R}^{u}=\frac{\left(M_{\tilde{u}}^{2}\right)_{23}}{\left(M_{\tilde{u}}\right)_{22}\left(M_{\tilde{u}}\right)_{33}} .
$$

In contrast to quantities like $\delta_{L L}^{u}$ or $\delta_{R L}^{u}$ where mixing with $\tilde{t}_{L}$ is considered, purely righthanded scenarios do not require a light bottom squark, and are hence not subject to strong constraints from direct sbottom searches [28, 53-55]. Quark flavour constraints are also avoided if only $\delta_{R R}^{u}$ insertions are considered. We note that although the mass insertion parameter $\delta_{L R}^{u}$ does not require a light sbottom and is poorly constrained by flavour physics, its effect on the stop decay width is suppressed relative to $\delta_{R R}^{u}$ by a factor of 16 due to hypercharges. Using the mass insertion parameter (4.1) as a template thus allows one to illustrate the maximal effects of flavour violation in stop decays. Choosing other possibilities would generically lead to stronger exclusions, once additional direct and/or indirect constraints are included.

In figure 8 , we show the dependence of $\operatorname{Br}\left(\tilde{t}_{1} \rightarrow t \tilde{\chi}_{1}^{0}\right)$ and $\operatorname{Br}\left(\tilde{t}_{1} \rightarrow W b \tilde{\chi}_{1}^{0}\right)$ on $\delta_{R R}^{u}$ for three different benchmark values of $\left(M_{\tilde{u}}\right)_{22}$. The left panel corresponds to $m_{\tilde{t}_{1}}=$ $500 \mathrm{GeV}$ and $m_{\tilde{\chi}_{1}^{0}}=200 \mathrm{GeV}$, i.e. a parameter point that lies in the heart of the kinematic region R1. Similarly, the curves in the right panel are based on the point $m_{\tilde{t}_{1}}=300 \mathrm{GeV}$ and $m_{\tilde{\chi}_{1}^{0}}=200 \mathrm{GeV}$, which is located in the kinematic region R2. Note that even though we parameterise our results in terms of (4.1), we perform an exact diagonalisation in our numerical analysis. One observes that to reduce $\operatorname{Br}\left(\tilde{t}_{1} \rightarrow t \tilde{\chi}_{1}^{0}\right)=1-\operatorname{Br}\left(\tilde{t}_{1} \rightarrow c \tilde{\chi}_{1}^{0}\right)$ from $100 \%$ to $90 \%$ requires large mass insertions $\delta_{R R}^{u} \in[0.6,0.8]$, while values of $\delta_{R R}^{u} \simeq 0.02$ are sufficient to suppress $\operatorname{Br}\left(\tilde{t}_{1} \rightarrow W b \tilde{\chi}_{1}^{0}\right)=1-\operatorname{Br}\left(\tilde{t}_{1} \rightarrow c \tilde{\chi}_{1}^{0}\right)$ by approximately $50 \%$. The strong $\delta_{R R}^{u}$-dependence of $\operatorname{Br}\left(\tilde{t}_{1} \rightarrow W b \tilde{\chi}_{1}^{0}\right)$ is a consequence of the fact that $\tilde{t}_{1} \rightarrow W b \tilde{\chi}_{1}^{0}$ is 

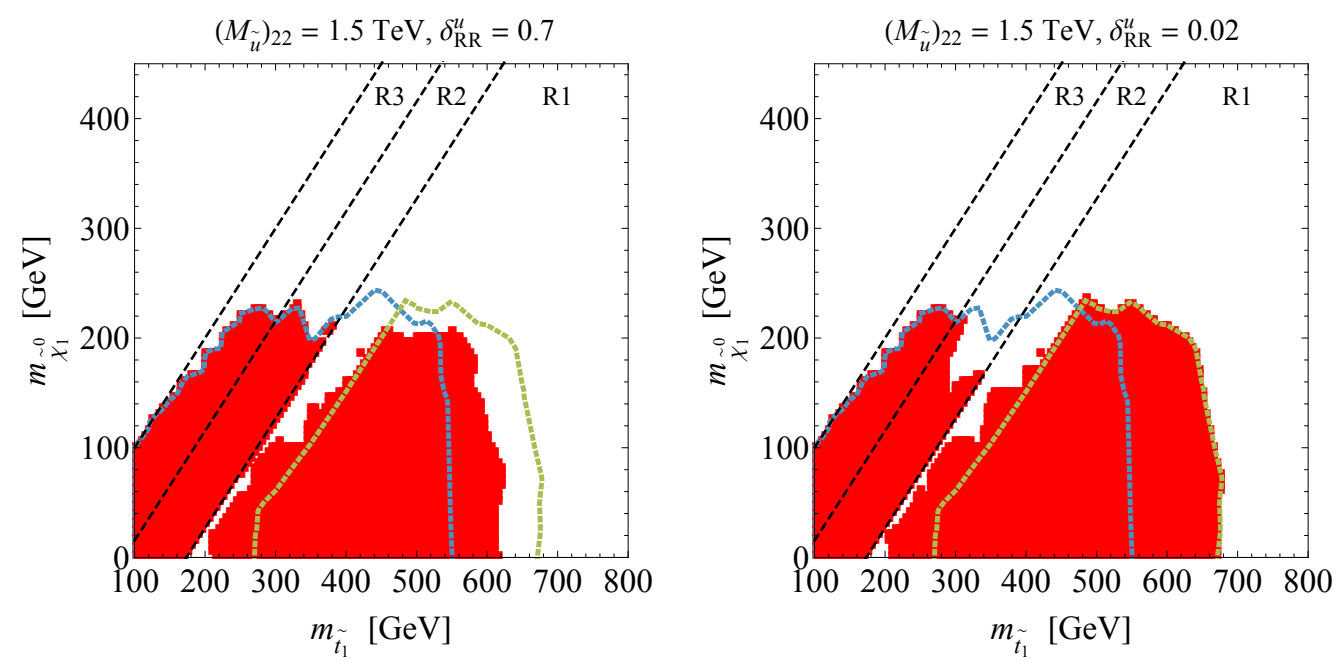

Figure 9. $95 \%$ CL exclusion regions in the $m_{\tilde{t}_{1}}-m_{\tilde{\chi}_{1}^{0}}$ plane for two representative $\tilde{t}_{R}-\tilde{c}_{R}$ mixing scenarios. The left (right) panel employs the parameters $\left(M_{\tilde{u}}\right)_{22}=1.5 \mathrm{TeV}$ and $\delta_{R R}^{u}=0.7$ $\left(\left(M_{\tilde{u}}\right)_{22}=1.5 \mathrm{TeV}\right.$ and $\left.\delta_{R R}^{u}=0.02\right)$. For comparison also the exclusion limits at 95\% CL following from the 1 lepton +4 jets $+1 b$-tag $+E_{T \text {,miss }}$ search [20] (green dotted curves) and the $2 c$-tags $+E_{T, \text { miss }}$ search [35] (blue dotted curves) are overlaid.

a three-body decay, whereas $\tilde{t}_{1} \rightarrow c \tilde{\chi}_{1}^{0}$ is a two-body process. In the case of $\tilde{t}_{1} \rightarrow t \tilde{\chi}_{1}^{0}$, the flavour-conserving decay mode is not phase-space suppressed and as a result a larger $\tilde{t}_{R^{-}}$ $\tilde{c}_{R}$ mixing angle is needed to obtain appreciable values of $\operatorname{Br}\left(\tilde{t}_{1} \rightarrow c \tilde{\chi}_{1}^{0}\right)$. In the kinematic region $\mathrm{R} 3, \tilde{t}_{1} \rightarrow c \tilde{\chi}_{1}^{0}$ is the dominant decay mode unless $\delta_{R R}^{u}$ is below $10^{-3}$, in which case the four-body mode $\tilde{t}_{1} \rightarrow b f f^{\prime} \tilde{\chi}_{1}^{0}$ becomes the main channel. In the following, we will not consider such small $\tilde{t}_{R}-\tilde{c}_{R}$ mixing angles and hence will employ $\operatorname{Br}\left(\tilde{t}_{1} \rightarrow c \tilde{\chi}_{1}^{0}\right)=100 \%$ in the whole region $\mathrm{R} 3$.

In the two panels of figure 9 , we show the results of the combined search strategies of sections 2 and 3 when applied to two representative scenarios of $\tilde{t}_{R}-\tilde{c}_{R}$ mixing. The left panel depicts the case of large mixing $\delta_{R R}^{u}=0.7$, while the right panel illustrates the case of small mixing $\delta_{R R}^{u}=0.02$. In both plots, we have fixed $\left(M_{\tilde{u}}\right)_{22}=1.5 \mathrm{TeV}$ and the red contours correspond to the regions in the $m_{\tilde{t}_{1}}-m_{\tilde{\chi}_{1}^{0}}$ plane that are excluded at $95 \%$ CL. To guide the eye, the 95\% CL exclusion limits obtained in [20] and [35] have been overlaid as green and blue dotted curves. Focusing our attention on the kinematic region R1, we see that for the choice $\delta_{R R}^{u}=0.7$, the limits on $m_{\tilde{t}_{1}}$ are about $50 \mathrm{GeV}$ weaker than the bound of $675 \mathrm{GeV}$ obtained in [20], which assumes no stop-scharm mixing. Hence, in this case, $\tilde{t}_{R}-\tilde{c}_{R}$ mixing only leads to an $8 \%$ reduction in the stop mass limits. On the other hand, for $\delta_{R R}^{u}=$ 0.02 our exclusion coincides with the limit of the 1 lepton +4 jets $+1 b$-tag $+E_{T, \text { miss }}$ search. These features are expected because in the first case one has $\operatorname{Br}\left(\tilde{t}_{1} \rightarrow t \tilde{\chi}_{1}^{0}\right) \in[70,80] \%$ in the parameter space of interest, while $\operatorname{Br}\left(\tilde{t}_{1} \rightarrow t \tilde{\chi}_{1}^{0}\right) \simeq 100 \%$ in the second case. In the kinematic region $\mathrm{R} 2$, one observes instead that for large $\tilde{t}_{R}-\tilde{c}_{R}$ mixing our bound resembles that of the analysis [35], while for small mixing the region in the $m_{\tilde{t}_{1}}-m_{\tilde{\chi}_{1}^{0}}$ plane around $m_{\tilde{t}_{1}}=300 \mathrm{GeV}$ and $m_{\tilde{\chi}_{1}^{0}}=200 \mathrm{GeV}$ remains allowed. These properties can 
be understood by realising that in the first case the lightest stop decays to almost $100 \%$ via $\tilde{t}_{1} \rightarrow c \tilde{\chi}_{1}^{0}$, while in the second case the decay mode $\tilde{t}_{1} \rightarrow W b \tilde{\chi}_{1}^{0}$ is dominant, in particular for values of the stop and LSP mass close to the kinematic boundary $m_{\tilde{t}_{1}}-m_{\tilde{\chi}_{1}^{0}}=m_{t}$. One furthermore notices, that in region R3 our exclusions match the 95\% CL bound from the $\tilde{c}_{1} \rightarrow c \tilde{\chi}_{1}^{0}$ analysis [35], since both our choices of $\delta_{R R}^{u}$ lead to $\operatorname{Br}\left(\tilde{t}_{1} \rightarrow c \tilde{\chi}_{1}^{0}\right)=100 \%$. The two scenarios of $\tilde{t}_{R}-\tilde{c}_{R}$ mixing that we have considered nicely illustrate our general finding that by combining various $E_{T \text {,miss }}$ search strategies, large regions in the $m_{\tilde{t}_{1}}-m_{\tilde{\chi}_{1}^{0}}$ plane can be excluded for arbitrary mass insertion parameters $\delta_{R R}^{u}$.

Notice that quark flavour observables leave the mass insertion parameter $\delta_{R R}^{u}$ essentially unconstrained. Although $\tilde{t}_{R}-\tilde{c}_{R}$ mixing will induce flavour-changing top-quark decays like $t \rightarrow c Z$ and $t \rightarrow c h$ at the one-loop level, the existing LHC Run I constraints on the relevant processes [56] are too loose to lead to any restriction. The mass insertion parameter $\delta_{R R}^{u}$ also modifies $B$-meson decays via chargino loops. However, the wino couples only to left-handed squarks and the Higgsino coupling to right-handed squarks is proportional to the corresponding Yukawa coupling, which is small in the case of the charm squark. As a result, corrections associated to the $\delta_{R R}^{u}$ mass insertion are strongly suppressed in processes like $B_{s} \rightarrow \mu^{+} \mu^{-}$and $B \rightarrow X_{s} \gamma$, making the constraints from stop searches derived above the only relevant restrictions on scenarios with purely right-handed stop-scharm mixing.

\section{Conclusions and outlook}

In this article, we have shown that allowing for non-minimal flavour violation in the upsquark sector of the MSSM can weaken the direct LHC bounds on the mass $m_{\tilde{t}_{1}}$ of the lightest stop. While large effects were found previously [31-33], we have demonstrated that a detailed numerical analysis which includes the recent ATLAS search for $\tilde{c}_{1} \rightarrow c \tilde{\chi}_{1}^{0}$ limits the possible impact of $\tilde{t}_{1} \rightarrow c \tilde{\chi}_{1}^{0}$ on $\tilde{t}_{1} \rightarrow c \tilde{\chi}_{1}^{0}$ and $\tilde{t}_{1} \rightarrow W b \tilde{\chi}_{1}^{0}$. The general idea is that although an enhanced $\tilde{t}_{1} \rightarrow c \tilde{\chi}_{1}^{0}$ decay rate decreases the branching ratios of $\tilde{t}_{1} \rightarrow t \tilde{\chi}_{1}^{0}$ and $\tilde{t}_{1} \rightarrow W b \tilde{\chi}_{1}^{0}$, the direct $\tilde{c}_{1} \rightarrow c \tilde{\chi}_{1}^{0}$ bounds become progressively more relevant, and as a result stop and scharm searches cannot be fully decoupled in the presence of upsquark mixing. By combining the different decay channels, we demonstrated that there are large regions in the $m_{\tilde{t}_{1}}-m_{\tilde{\chi}_{1}^{0}}$ plane which are disfavoured by LHC Run I searches, independently of the amount of stop-scharm mixing. In particular, we find a lower limit of $m_{\tilde{t}_{1}}>530 \mathrm{GeV}$ at $95 \% \mathrm{CL}$ for LSP masses $m_{\tilde{\chi}_{1}^{0}} \lesssim 100 \mathrm{GeV}$. This finding agrees with [34], generalising it to the case of a neutralino with non-zero mass. Our bound of $530 \mathrm{GeV}$ is only about $35 \%$ weaker than the limit of $715 \mathrm{GeV}$ that ATLAS obtains from a combination of LHC Run I data [21] assuming a $\tilde{t}_{1} \rightarrow t \tilde{\chi}_{1}^{0}$ branching ratio of $100 \%$. Stringent exclusion limits can also be derived for all other considered decay scenarios. We have illustrated this point by studying MSSM scenarios with a bino-like LSP and non-zero $\tilde{t}_{R}-\tilde{c}_{R}$ mixing. The two representative cases of the mass insertion parameter $\delta_{R R}^{u}$ that we have considered are left unconstrained by quark flavour observables, but by combining various direct $E_{T, \text { miss }}$ searches, stringent exclusions in the $m_{\tilde{t}_{1}}-m_{\tilde{\chi}_{1}^{0}}$ plane can be derived. 
In LHC Run II and beyond, the ATLAS and CMS collaborations are expected to provide new results on stop searches with a siginificantly improved reach in the $m_{\tilde{t}_{1}}-m_{\tilde{\chi}_{1}^{0}}$ plane. Improvements in the sensitivity to stops will not only be due to the increase in the centre-of-mass energy, but is also likely to arise from new analysis strategies or technical developments. For instance, ATLAS has recently installed [57] a new subdetector called Insertable B-Layer or IBL [58]. This new inner pixel layer should allow to improve the $c$-tagging capabilities of ATLAS and thus pave the way to look for processes like $\tilde{c}_{1} \rightarrow c \tilde{\chi}_{1}^{0}$ and $\tilde{t}_{1} \rightarrow c \tilde{\chi}_{1}^{0}$ in a more efficient fashion. The complementarity and synergy between the different stop decay channels that exists in the presence of flavour mixing is therefore expected to become phenomenologically even more relevant at later phases of the LHC physics programme.

\section{Acknowledgments}

We thank William Kalderon for useful discussions concerning the ATLAS search for scalar charm quarks [35]. The work of AC was supported by a Marie Curie Intra-European Fellowship of the European Community's 7th Framework Programme (contract number PIEF-GA-2012-326948) and by an Ambizione Grant of the Swiss National Science Foundation. UH acknowledges the hospitality and support of the CERN theory division. He also would like to thank the KITP in Santa Barbara for hospitality and acknowledges that this research was supported in part by the National Science Foundation under Grant No. NSF PHY11-25915. LCT is supported by the Swiss National Science Foundation.

\section{A Event generation}

Our event generation has been performed at leading order with MadGraph5_aMCNLO [59] starting from a customised version of the implementation of coloured scalar pair production presented in [60] and utilises NNPDF2.3 parton distribution functions [61]. The simulated parton-level events were showered with PYTHIA 6 [62] and analysed with the publicly available code CheckMATE [63], which relies on DELPHES 3 [64] as a fast detector simulation. In order to be able to distinguish charm-quark jets from both bottom-quark and light-flavoured jets, we have implemented the JetFitterCharm algorithm described in [42] into DELPHES 3. In all our analyses jets were clustered with Fast Jet [65] with the anti- $k_{t}$ algorithm [66] as the standard jet finder.

The efficiency maps presented in figures 2 and 6 have been obtained by simulating 138 different signal points that fall into the kinematic regions R1 and R2. The actual mapping in the $m_{\tilde{t}_{1}}-m_{\tilde{\chi}_{1}^{0}}$ plane can be found in $[67,68]$. For each signal point and all relevant final states, $10^{5}$ partonic events have been generated, showered and passed through the fast detector simulation and an analysis containing the selection requirements corresponding to the individual searches described in sections 2 and 3. The efficiency maps are then obtained by considering the SR that provides the best exclusion limit for a given point in the $m_{\tilde{t}_{1}}-m_{\tilde{\chi}_{1}^{0}}$ plane. 
Open Access. This article is distributed under the terms of the Creative Commons Attribution License (CC-BY 4.0), which permits any use, distribution and reproduction in any medium, provided the original author(s) and source are credited.

\section{References}

[1] M. Dine, R.G. Leigh and A. Kagan, Flavor symmetries and the problem of squark degeneracy, Phys. Rev. D 48 (1993) 4269 [hep-ph/9304299] [INSPIRE].

[2] S. Dimopoulos and G.F. Giudice, Naturalness constraints in supersymmetric theories with nonuniversal soft terms, Phys. Lett. B 357 (1995) 573 [hep-ph/9507282] [INSPIRE].

[3] A. Pomarol and D. Tommasini, Horizontal symmetries for the supersymmetric flavor problem, Nucl. Phys. B 466 (1996) 3 [hep-ph/9507462] [INSPIRE].

[4] A.G. Cohen, D.B. Kaplan and A.E. Nelson, The More minimal supersymmetric Standard Model, Phys. Lett. B 388 (1996) 588 [hep-ph/9607394] [InSPIRE].

[5] R. Kitano and Y. Nomura, Supersymmetry, naturalness and signatures at the LHC, Phys. Rev. D 73 (2006) 095004 [hep-ph/0602096] [INSPIRE].

[6] C. Brust, A. Katz, S. Lawrence and R. Sundrum, SUSY, the Third Generation and the LHC, JHEP 03 (2012) 103 [arXiv:1110.6670] [InSPIRE].

[7] M. Papucci, J.T. Ruderman and A. Weiler, Natural SUSY Endures, JHEP 09 (2012) 035 [arXiv:1110.6926] [INSPIRE].

[8] P. Batra, A. Delgado, D.E. Kaplan and T.M.P. Tait, The Higgs mass bound in gauge extensions of the minimal supersymmetric standard model, JHEP 02 (2004) 043 [hep-ph/0309149] [INSPIRE].

[9] L.J. Hall, D. Pinner and J.T. Ruderman, A Natural SUSY Higgs Near 126 GeV, JHEP 04 (2012) 131 [arXiv:1112.2703] [INSPIRE].

[10] S.P. Martin, A Supersymmetry primer, hep-ph/9709356 [INSPIRE].

[11] C. Boehm, A. Djouadi and M. Drees, Light scalar top quarks and supersymmetric dark matter, Phys. Rev. D 62 (2000) 035012 [hep-ph/9911496] [InSPIRE].

[12] J.R. Ellis, K.A. Olive and Y. Santoso, Calculations of neutralino stop coannihilation in the CMSSM, Astropart. Phys. 18 (2003) 395 [hep-ph/0112113] [INSPIRE].

[13] M. Carena, M. Quirós and C.E.M. Wagner, Opening the window for electroweak baryogenesis, Phys. Lett. B 380 (1996) 81 [hep-ph/9603420] [INSPIRE].

[14] J.R. Espinosa, Dominant two loop corrections to the MSSM finite temperature effective potential, Nucl. Phys. B 475 (1996) 273 [hep-ph/9604320] [INSPIRE].

[15] D. Delepine, J.M. Gerard, R. Gonzalez Felipe and J. Weyers, A Light stop and electroweak baryogenesis, Phys. Lett. B 386 (1996) 183 [hep-ph/9604440] [INSPIRE].

[16] ATLAS collaboration, Summary plots from the ATLAS Supersymmetry physics group, https://atlas.web.cern.ch/[...]/index.html\#ATLAS_SUSY_Summary.

[17] CMS collaboration, Summary of comparison plots in simplified models spectra for the $8 \mathrm{TeV}$ dataset, https://twiki.cern.ch/twiki/bin/view/CMSPublic/SUSYSMSSummaryPlots8TeV. 
[18] ATLAS collaboration, Search for direct top-squark pair production in final states with two leptons in pp collisions at $\sqrt{s}=8 \mathrm{TeV}$ with the ATLAS detector, JHEP 06 (2014) 124 [arXiv: 1403.4853] [INSPIRE].

[19] ATLAS collaboration, Search for direct pair production of the top squark in all-hadronic final states in proton-proton collisions at $\sqrt{s}=8 \mathrm{TeV}$ with the ATLAS detector, JHEP 09 (2014) 015 [arXiv: 1406.1122] [INSPIRE].

[20] ATLAS collaboration, Search for top squark pair production in final states with one isolated lepton, jets and missing transverse momentum in $\sqrt{s}=8 \mathrm{TeV}$ pp collisions with the ATLAS detector, JHEP 11 (2014) 118 [arXiv:1407.0583] [INSPIRE].

[21] ATLAS collaboration, ATLAS Run 1 searches for direct pair production of third-generation squarks at the Large Hadron Collider, Eur. Phys. J. C 75 (2015) 510 [arXiv:1506.08616] [INSPIRE].

[22] ATLAS collaboration, Search for top squarks in final states with one isolated lepton, jets and missing transverse momentum in $\sqrt{s}=13 \mathrm{TeV}$ pp collisions of ATLAS data, ATLAS-CONF-2016-007 (2016).

[23] ATLAS collaboration, Search for direct top squark pair production in final states with two leptons in $\sqrt{s}=13$ TeV pp collisions using $3.2 \mathrm{fb}^{-1}$ of ATLAS data, ATLAS-CONF-2016-009 (2016).

[24] CMS collaboration, Search for top-squark pair production in the single-lepton final state in pp collisions at $\sqrt{s}=8 \mathrm{TeV}$, Eur. Phys. J. C 73 (2013) 2677 [arXiv:1308.1586] [InSPIRE].

[25] CMS collaboration, Search for top squarks in multijet events with large missing momentum in proton-proton collisions at $8 \mathrm{TeV}$,

http://cds.cern.ch/record/1635353/files/SUS-13-015-pas.pdf.

[26] CMS collaboration, Exclusion limits on gluino and top-squark pair production in natural SUSY scenarios with inclusive razor and exclusive single-lepton searches at $8 \mathrm{TeV}$, https://cds.cern.ch/record/1745586/files/SUS-14-011-pas.pdf.

[27] CMS collaboration, Search for direct top squark pair production in the single lepton final state at $\sqrt{s}=13 \mathrm{TeV}$ https://cds.cern.ch/record/2139650/files/SUS-16-002-pas.pdf.

[28] CMS collaboration, Further SUSY Simplified Model interpretations for Moriond 2016, https://cds.cern.ch/record/2140312/files/SUS-16-004-pas.pdf.

[29] B. Fuks, B. Herrmann and M. Klasen, Flavour Violation in Gauge-Mediated Supersymmetry Breaking Models: Experimental Constraints and Phenomenology at the LHC, Nucl. Phys. B 810 (2009) 266 [arXiv:0808.1104] [INSPIRE].

[30] T. Hurth and W. Porod, Flavour violating squark and gluino decays, JHEP 08 (2009) 087 [arXiv:0904.4574] [INSPIRE].

[31] A. Bartl, H. Eberl, E. Ginina et al., Flavor violating bosonic squark decays at LHC, Int. J. Mod. Phys. A 29 (2014) 1450035 [arXiv:1212.4688] [INSPIRE].

[32] M. Blanke, G.F. Giudice, P. Paradisi, G. Perez and J. Zupan, Flavoured Naturalness, JHEP 06 (2013) 022 [arXiv: 1302.7232] [INSPIRE].

[33] P. Agrawal and C. Frugiuele, Mixing stops at the LHC, JHEP 01 (2014) 115 [arXiv: 1304.3068] [INSPIRE]. 
[34] M. Blanke, B. Fuks, I. Galon and G. Perez, Gluino Meets Flavored Naturalness, JHEP 04 (2016) 044 [arXiv : 1512.03813] [INSPIRE].

[35] ATLAS collaboration, Search for Scalar Charm Quark Pair Production in pp Collisions at $\sqrt{s}=8 \mathrm{TeV}$ with the ATLAS Detector, Phys. Rev. Lett. 114 (2015) 161801 [arXiv: 1501.01325] [INSPIRE].

[36] R. Gröber, M. Mühlleitner, E. Popenda and A. Wlotzka, Light stop decays into Wb $\tilde{\chi}_{1}^{0}$ near the kinematic threshold, Phys. Lett. B 747 (2015) 144 [arXiv:1502.05935] [InSPIRE].

[37] ATLAS collaboration, Search for pair-produced third-generation squarks decaying via charm quarks or in compressed supersymmetric scenarios in pp collisions at $\sqrt{s}=8 \mathrm{TeV}$ with the ATLAS detector, Phys. Rev. D 90 (2014) 052008 [arXiv: 1407.0608] [InSPIRE].

[38] R. Gröber, M.M. Mühlleitner, E. Popenda and A. Wlotzka, Light Stop Decays: Implications for LHC Searches, Eur. Phys. J. C 75 (2015) 420 [arXiv: 1408.4662] [INSPIRE].

[39] Y. Nir and N. Seiberg, Should squarks be degenerate?, Phys. Lett. B 309 (1993) 337 [hep-ph/9304307] [INSPIRE].

[40] R. Mahbubani, M. Papucci, G. Perez, J.T. Ruderman and A. Weiler, Light Nondegenerate Squarks at the LHC, Phys. Rev. Lett. 110 (2013) 151804 [arXiv:1212.3328] [INSPIRE].

[41] A. Crivellin and M. Davidkov, Do squarks have to be degenerate? Constraining the mass splitting with Kaon and D mixing, Phys. Rev. D 81 (2010) 095004 [arXiv:1002.2653] [INSPIRE].

[42] ATLAS collaboration, Performance and Calibration of the JetFitterCharm Algorithm for c-Jet Identification, ATL-PHYS-PUB-2015-001 (2015).

[43] G. Polesello and D.R. Tovey, Supersymmetric particle mass measurement with the boost-corrected contransverse mass, JHEP 03 (2010) 030 [arXiv:0910.0174] [INSPIRE].

[44] ATLAS collaboration, Calibrating the b-Tag Efficiency and Mistag Rate in $35 \mathrm{pb}^{-1}$ of Data with the ATLAS Detector, ATLAS-CONF-2011-089 (2011).

[45] ATLAS collaboration, Commissioning of the ATLAS high-performance b-tagging algorithms in the $7 \mathrm{TeV}$ collision data, ATLAS-CONF-2011-102 (2011).

[46] H.-C. Cheng and Z. Han, Minimal Kinematic Constraints and $m_{T 2}$, JHEP 12 (2008) 063 [arXiv:0810.5178] [INSPIRE].

[47] A.J. Barr, B. Gripaios and C.G. Lester, Transverse masses and kinematic constraints: from the boundary to the crease, JHEP 11 (2009) 096 [arXiv:0908.3779] [INSPIRE].

[48] P. Konar, K. Kong, K.T. Matchev and M. Park, Dark Matter Particle Spectroscopy at the LHC: Generalizing $m_{T 2}$ to Asymmetric Event Topologies, JHEP 04 (2010) 086 [arXiv: 0911.4126] [INSPIRE].

[49] Y. Bai, H.-C. Cheng, J. Gallicchio and J. Gu, Stop the Top Background of the Stop Search, JHEP 07 (2012) 110 [arXiv: 1203.4813] [inSPIRE].

[50] C.G. Lester and D.J. Summers, Measuring masses of semiinvisibly decaying particles pair produced at hadron colliders, Phys. Lett. B 463 (1999) 99 [hep-ph/9906349] [INSPIRE].

[51] A. Barr, C. Lester and P. Stephens, $m_{T 2}$ : The Truth behind the glamour, J. Phys. G 29 (2003) 2343 [hep-ph/0304226] [INSPIRE]. 
[52] F. Gabbiani, E. Gabrielli, A. Masiero and L. Silvestrini, A Complete analysis of FCNC and CP constraints in general SUSY extensions of the standard model, Nucl. Phys. B 477 (1996) 321 [hep-ph/9604387] [INSPIRE].

[53] ATLAS collaboration, Search for direct third-generation squark pair production in final states with missing transverse momentum and two b-jets in $\sqrt{s}=8 \mathrm{TeV}$ pp collisions with the ATLAS detector, JHEP 10 (2013) 189 [arXiv:1308.2631] [INSPIRE].

[54] CMS collaboration, Search for direct production of bottom squark pairs, http://cds.cern.ch/record/1693164/files/SUS-13-018-pas.pdf.

[55] ATLAS collaboration, Search for Bottom Squark Pair Production with the ATLAS Detector in proton-proton Collisions at $\sqrt{s}=13 \mathrm{TeV}$, ATLAS-CONF-2015-066 (2015).

[56] A. Loginov, ATLAS + CMS top production and properties: run 1 legacy, in proceedings of 50th Rencontres de Moriond Electroweak interactions and unified theories, 2015, p. 149, http://inspirehep.net/record/1423200/files/Pages_from_C15-03-14_149.pdf.

[57] A.E. Phoboo and C.O'Luanaigh, A new subdetector for ATLAS, http://cds.cern.ch/record/1998718.

[58] M. Capeans, G. Darbo, K. Einsweiller, M. Elsing, T. Flick, et al., ATLAS Insertable B-Layer Technical Design Report, https://cds.cern.ch/record/1291633.

[59] J. Alwall, R. Frederix, S. Frixione et al., The automated computation of tree-level and next-to-leading order differential cross sections and their matching to parton shower simulations, JHEP 07 (2014) 079 [arXiv: 1405.0301] [INSPIRE].

[60] C. Degrande, B. Fuks, V. Hirschi, J. Proudom and H.-S. Shao, Automated next-to-leading order predictions for new physics at the LHC: the case of colored scalar pair production, Phys. Rev. D 91 (2015) 094005 [arXiv:1412.5589] [InSPIRE].

[61] R.D. Ball et al., Parton distributions with LHC data, Nucl. Phys. B 867 (2013) 244 [arXiv:1207.1303] [INSPIRE].

[62] T. Sjöstrand, S. Mrenna and P.Z. Skands, PYTHIA 6.4 Physics and Manual, JHEP 05 (2006) 026 [hep-ph/0603175] [INSPIRE].

[63] M. Drees, H. Dreiner, D. Schmeier, J. Tattersall and J.S. Kim, CheckMATE: Confronting your Favourite New Physics Model with LHC Data, Comput. Phys. Commun. 187 (2015) 227 [arXiv: 1312.2591] [INSPIRE].

[64] DELPHES 3 collaboration, J. de Favereau et al., DELPHES 3, A modular framework for fast simulation of a generic collider experiment, JHEP 02 (2014) 057 [arXiv:1307.6346] [INSPIRE].

[65] M. Cacciari, G.P. Salam and G. Soyez, FastJet User Manual, Eur. Phys. J. C 72 (2012) 1896 [arXiv: 1111.6097] [INSPIRE].

[66] M. Cacciari, G.P. Salam and G. Soyez, The Anti-k $k_{t}$ jet clustering algorithm, JHEP 04 (2008) 063 [arXiv: 0802.1189] [INSPIRE].

[67] HepData, AAD 2014 - Search for top squark pair production in final states with one isolated lepton, jets, and missing transverse momentum in $\sqrt{s}=8 \mathrm{TeV}$ pp collisions with the ATLAS detector, http://hepdata.cedar.ac.uk/view/ins1304456/d11.

[68] HepData, AAD 2015 - Search for Scalar Charm Quark Pair Production in pp Collisions at $\sqrt{s}=8 \mathrm{TeV}$ with the ATLAS Detector, http://hepdata.cedar.ac.uk/view/ins1337472/d5;jsessionid=1ldlomhkyk0ac. 